\title{
Retour sur la session de printemps des Chambres fédérales
}

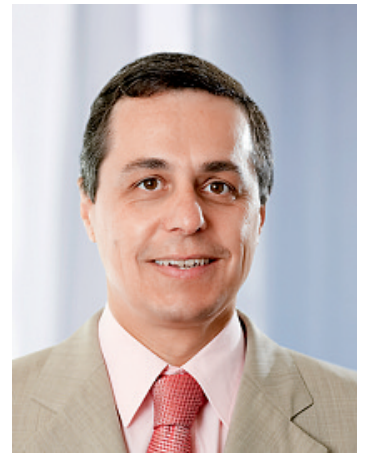

Ignazio Cassis
La santé n'a pas été un sujet central de la dernière session du Parlement suisse. Les thèmes dominants ont été ceux de la crise financière, de la question fiscale ainsi que de la pression internationale sur notre secret bancaire. Par contre des thèmes de recherche ont été débattus:

\section{Recherche sur l'être humain. \\ Article constitutionnel (07.072)}

Actuellement, la compétence en matière de recherche sur l'être humain n'est pas définie au plan fédéral et il n'existe pas de loi en la matière. Le projet d'un nouvel article constitutionnel 118 a et d'une loi sur la recherche sur l'être humain veut donner à la Confédération la compétence de régler l'ensemble de ce domaine. L'idée essentielle est de préserver la dignité et la personnalité de l'être humain tout en veillant à la liberté de la recherche et à l'importance de cette dernière pour la santé et la société. Au Conseil national, nous avons formulé cet article plus largement qu'une simple norme de compétence en mettant l'accent sur la recherche biomédicale. Le projet retourne maintenant aux Etats pour l'élimination des divergences.

\section{Convention sur les Droits de I'Homme} et la biomédecine. Protocole additionnel (08.064) Nous avons ratifié le protocole additionnel qui précise les règles de la Convention sur les Droits de l'Homme et la biomédecine dans le domaine de la médecine de la transplantation. Sauf trois aspects relatifs aux dons par des personnes vivantes, ce texte concorde avec la loi du 8 octobre 2004 sur la transplantation. Ces trois aspects ont donné lieu à la formulation de réserves pour la ratification.

\section{Loi sur la recherche. Révision partielle (08.079)}

La Suisse fait partie au plan mondial des Etats affichant la capacité d'innovation la plus élevée. Elle ne peut maintenir cette position que si les entreprises restent en mesure de développer des produits innovants et de les commercialiser avec succès. C'est là qu'intervient l'encouragement de l'innovation par la Confédération, destiné à jeter des ponts entre économie et science. A raison de 100 millions de francs par an, la Confédération soutient le développement de nouveaux produits, le transfert de savoir des hautes écoles dans l'économie et la création de jeunes entreprises. Cet encouragement a aussi contribué au déve- loppement de la recherche appliquée dans les HES. La révision partielle de la loi conférera à la Commission pour la technologie et l'innovation un cahier des charges et des compétences décisionnelles propres.

\section{Moyens et appareils médicaux. Potentiel d'économies}

Notre Conseil a approuvé une motion de Bea Heim (05.3522) et une autre de Ruth Humbel (05.3523) chargeant le DFI de faire pression sur les prix des moyens et appareils médicaux, se référant à la loi sur les cartels. Cette disposition permettrait d'éviter une éventuelle entente sur les prix.

\section{Prestations LAMal et allocations AVS/AI pour impotents. Prévoir un outil d'évaluation unique des soins requis}

Notre Conseil a approuvé la motion de Ruth Humbel (04.3719) qui demande au Conseil fédéral que les prestations pour soins LAMal et les allocations pour impotents LAVS et LAI soient déterminées au moyen d'un instrument unique d'évaluation des soins requis. Une simplification de la bureaucratie.

Gel des admissions. Solution transitoire (09.400) Après le fiasco de décembre au Conseil de Etat, qui n'a pas réussi à trouver une solution pour la suite du régime transitoire au gel des cabinets, la question à été reprise par la CSSS-N, qui en a fait une initiative parlementaire. Elle prévoit une prolongation du gel jusqu'à fin 2011, le gel du secteur ambulatoire des hôpitaux et la fin du gel pour les médecins de premiers recours, qui dès le $1^{\text {er }}$ janvier 2010 pourraient ainsi retrouver la liberté d'ouvrir des cabinets en régime LAMal.

Avec sa motion 08.3929 Erika Forster a essayé - sans succès de convaincre le Conseil des Etats (CdE) de trouver une solution alternative (modèle duale). Anita Fetz, pour sa part, a soutenu au CdE - également sans succès - sa motion 08.4048, visant aussi à introduire un autre modèle (modèle FMH-CDS). Le CdE a décidé de laisser cette tâche au Conseil national, qui a déjà élaboré une solution transitoire (09.400). En même temps la CSSS-N démarre en avril les travaux portant sur la réforme globale de la médecine ambulatoire. Ces travaux devraient aboutir à une réforme complète dès le $1^{\text {er }}$ janvier 2012. Affaire à suivre ...

Ignazio Cassis, vice-président FMH, Conseiller national

PS. Pour plus d'informations je vous invite à surfer sur le site www.parlement.ch et à introduire les petits numéros (p.ex. 09.400) dans la case «recherche». Bon voyage! 